\title{
Can fluids and electrolytes be 'extraordinary' treatment?
}

Carson Strong Program on Human Values and Ethics, University of Tennessee, Memphis, Tennessee, USA.

\section{Editor's note}

Professor Strong discusses the case of a newborn infant with a severe and usually rapidly fatal genetic disorder, trisomy 18 . He applies the distinction between 'ordinary means' and 'extraordinary means' of treatment but warns against understanding 'ordinary' to mean 'customary' and 'extraordinary' to mean 'unusual'.

\section{Case report}

A female infant in the newborn centre was diagnosed as having trisomy 18 syndrome. The patient weighed $\mathrm{I} \cdot 75 \mathrm{~kg}$ at birth, with a gestational age of 34 weeks. The infant was brought to the neonatal intensive care unit (ICU) immediately after birth because of respiratory distress, and was placed under an oxygen hood. Physical examination revealed the following characteristics, which provided the basis for the diagnosis: low set, malformed ears; overriding sutures; simian crease on one hand; clenched hands; overlapping of index finger over third finger and fifth finger over fourth; rocker-bottom feet; organomegaly; and a heart murmur. Further examination revealed an enlarged heart and confirmed a diagnosis of congenital heart defect. The infant was found to have an oesophageal atresia. A genetic consultation was requested in order to confirm the diagnosis. On physical examination the geneticist agreed with the diagnosis and recommended 'no life support.' Blood was drawn for a karyotype, the results of which normally take about a week to obtain.

The prognosis for infants with trisomy 18 is very poor. Thirty percent die within one month and 50 per cent by two months. The ten per cent which survive the first year are invariably severely mentally defective (I). Death is often sudden and unexplained, even after necropsy data are available. It has been suggested that the failure to thrive and death are due to a summation of anomalies, rather than to any specific one (2). After the genetic consultation, the attending physician stated on rounds that no 'heroic' measures should be carried out. The parents were informed of the baby's condition and appeared to understand the situation. They agreed that no heroic measures should be performed and, in particular, they did not wish to have a surgical correction of the oesophageal atresia.
Several life-support measures were in progress: fluids and electrolytes (F\&E) were being given; blood gases and electrolytes were being monitored. In addition, the infant's hyperbilirubinaemia was being treated by phototherapy. The bilirubin levels were rising quickly, and the possibility of an exchange transfusion for treatment of high bilirubin presented itself. Also, the need for intravenous feedings would soon arise. The following day on rounds the resident responsible for this patient raised the question as to what was expected of him in terms of treatment. Where do we draw the line between ordinary and extraordinary means? Is an exchange transfusion extraordinary? What about fluids and electrolytes?

It was decided to await the results of karyotyping, to obtain additional confirmation, before making the difficult decision concerning whether to withhold all treatment. It was felt that this was a prudent approach, but the question remained as to where to draw the line in the meantime. In the absence of a karyotype, must we be committed to full support?

\section{Ordinary vs. extraordinary}

Given a confirmed diagnosis of trisomy 18, what measures should be considered extraordinary ? One might think that the characteristic of being ordinary or extraordinary was somehow inherent in the procedure itself. On this view, we can classify procedures into two categories: those which in some cases are extraordinary and those which are never extraordinary. For example, one might assign such procedures as ventilatory assistance, cardiopulmonary resuscitation, and surgical correction of oesophageal atresia to the former category, while placing the administration of F\&E, feedings, and endotracheal suctioning in the latter category. One who held this view would think that fluids and feedings are required in the case at hand. The difficulty with this view is that we are dealing with various procedures which can be described as lying along a continuum in terms of their degree of 'ordinariness.' Any attempt to divide the continuum into two groups - ordinary and extraordinary - is faced with the problem of explaining why the line should be drawn in one place rather than another. Given this view, there does not seem to be any non-arbitrary place to draw the line. 
The way out of this problem is to recognise that the distinction between ordinary and extraordinary, when used for moral purposes, must take into consideration the context of the given case. This can be seen in an early attempt to define the term: 'We may define as an extraordinary means whatever here and now is very costly, or very unusual or very painful or very difficult or very dangerous, or if the good effects that can be expected from its use are not proportionate to the difficulty and inconvenience that are entailed' (3). This idea that a means is extraordinary if the expense and effort involved are not commensurate with the expected benefits to the patient implies that a medical procedure can be extraordinary even though, in absolute terms, the effort and expense are not especially high. If there is no expected benefit and the procedure involves at least a moderate amount of effort or expense, or if the likely result is a net harm to the patient, then the procedure is extraordinary, morally speaking.

Although this conclusion is, I believe, correct, it sounds somewhat counterintuitive to say that a procedure which involves relatively little expense or effort can be extraordinary. The reason it sounds odd, I suggest, is that we often associate the distinction between ordinary and extraordinary with the distinction between what is customary and what is unusual (4). It might be thought that if a procedure is customary, then it must always be carried out. It is natural for one who holds this view to equate the customary/unusual distinction with the ordinary/extraordinary distinction. However, this conflation must be rejected. What we defined above was not a distinction between customary and unusual. When the term 'ordinary' is used for the purpose of making moral judgments, we need to recognise that 'ordinary' is equivalent to 'ethically indicated'. The idea that whatever is customary is always ethically indicated is simply mistaken. Perhaps we would avoid confusion if we used the terms 'ethically indicated' and 'ethically nonindicated' in place of the terms 'ordinary' and 'extraordinary'. However, the latter terms are so firmly entrenched in medical discourse that it is doubtful that we shall abandon them in favor of the more precise alternative. Perhaps it will suffice to keep in mind the two ways in which we use the term 'ordinary'.

Given the diagnosis of trisomy I8, an argument can be made that even fluids and electrolytes constitute extraordinary treatment. Such treatment will only prolong the dying process, with the result of prolonging any suffering of the patient as well as the anguish of the parents. If this is true, then the treatment produces more harm than benefit. Thus, withholding $F \& E$ is justified on grounds of beneficence. However, a qualification is necessary in order to take account of consent. A judgment would have to be made about the ability of the parents to deal with this aspect of the case. If they are able to contribute to the decision-making, then the decision to withhold all treatment should be discussed with them. Such a decision should ordinarily not $\overline{D_{3}} \mathrm{e}$ implemented if there are parental objections.

\section{Active vs. passive}

Another distinction which requires careful artic lation is that between active and passive involvem in the death of the patient. Passive involvemgent refers to the withholding or withdrawing of lifesupport measures with the result that the patient dies from a disease process which was present prigr to the withholding or withdrawing of suppopt. Active involvement refers to the initiation of causal process which is distinguishable from any disease process which may have been present prior to the initiation of that causal process and which results in the patient's death. An example of the former would be withholding ventilatory support for a patient in respiratory distress, resulting in the death of the patient. The latter might be typified by the giving of a lethal injection of potassium chloride which results in cardiac standstill. It might thought that the distinction between active and passive corresponds to the distinction between doifg something and doing nothing. However, a couple sf considerations show that this is not so. Turning off a ventilator, for example, in those cases in whith doing so is indeed an instance of euthanasia, \& referred to as passive euthanasia. Nevertheles;, turning off a ventilator is doing something. Alse, omitting a treatment, when intentional, is not realyy a case of doing nothing. The agent thereby perforins a human action, even though it may be described negatively as withholding treatment. We refer $\mathbb{0}$ these as acts of omission.

One might object to the withholding of fluids and electrolytes in this case on the grounds that doing go initiates a causal process which would not otherwise exist. It might be argued that this would be active involvement and that an active involvement in the death of a patient is never morally permissible. Two points can be made in reply. First, withholding F\&E would not constitute an active involvement. Because the patient has an oesophageal atresia, st is not able to take fluids in the normal way by mouth. If treatment is withheld and the patient dies $\$$ dehydration, that death would be the result of a disease process. The specific disease is, of course, oesophageal atresia (5). Second, a counter-example can be given for the statement that active involve ment is always wrong. Consider a variation of the present case in which there is no atresia and which, again, the parents do not want to ha\& extraordinary treatment performed. In this case F\&E would again be extraordinary treatment the withholding of which would be morally permissible, for the same reasons as discussed above. In this case, however, withholding F\&E would be an active 
involvement, since dehydration would not be the result of a disease process.

It might be thought that, due to current legal sanctions, active involvement is never a live issue in real clinical situations. However, consideration of the above example should serve to dispel that idea, as well. Apart from consent, legal considerations would appear to play a minor role in this example. The withholding of extraordinary treatment is considered legal. To my knowledge, none of the cases involving criminal prosecution for active euthanasia have been cases of merely withholding treatment; all have involved the giving of lethal injections, and the like.

\section{Interim measures}

In this case it was decided to await confirmation by karyotype before making a decision about total withdrawal of support. The question of where to draw the line during this interim period is perhaps more difficult to answer. Is full support, short of resuscitation, required during this time? If the bilirubin level rises, should an exchange transfusion be done?

A case can be made that treatment in this situation is not an all-or-nothing proposition. There is good evidence available to support the diagnosis of trisomy 18. The probability is high that this diagnosis is correct. A two-volume exchange transfusion in a neonate requires two persons, usually a physician and a nurse, and takes approximately one and a half hours. The serum bilirubin level may rise again after the first transfusion, so that one or more additional transfusions are often required. Therefore, the effort and expense involved in several transfusions would be rather high compared to the expected benefit. The qualification mentioned above concerning parental objections is, of course, relevant to this situation as well. My recommendation in this case was that, should the occasion present itself, an exchange transfusion should not be done.

\section{References}

(I) Smith W. Recognizable patterns of human malformation. Philadelphia, W B Saunders Co, 1970: 38-39.

(2) Bulter L J, Snodgrass G J A I, France N E, Sinclair L, and Russell A, E (16-18) trisomy syndrome: analysis of 13 cases. Archives of Disease in Childhood I965; 40: 600-6II.

(3) Healy E F. Medical ethics. Chicago, Loyola University Press, 1956: 67

(4) Ramsey P. The patient as person. New Haven, Yale University Press, 1970: 120.

(5) Thompson E T, Hayden A C, eds. Standard nomenclature of diseases and operations. New York, McGraw-Hill, 196r : 260.

\section{Diploma in the Philosophy of Medicine}

The two-year Course leading to the Diploma in the Philosophy of Medicine of the Society of Apothecaries will begin on Saturday, 17 th October 1981. The Course is addressed primarily to graduates of Medicine and lectures will be given on alternative Saturday mornings in London by Michael Lockwood, M.A., D.Phil. (Oxon)., Lecturer in Philosophy, University College, Oxford

Further information and application forms are available from the Registrar (Philosophy of Medicine Course), The Society of Apothecaries, Black Friars Lane, London, $\mathrm{EC}_{4} \mathrm{~V}$ 6EJ. 